\title{
La compréhension et l'expression en environnement multimédia en présentiel et en autonomie guidée
}

A piece of cake ou encore $\mathrm{C} 2=\mathrm{E} / \mathrm{M}$, théorisation d'une pratique

\section{Alain Ginet}

\section{OpenEdition}

\section{Journals}

Édition électronique

URL : http://journals.openedition.org/asp/3947

DOI : 10.4000/asp.3947

ISSN : 2108-6354

Éditeur

Groupe d'étude et de recherche en anglais de spécialité

Édition imprimée

Date de publication : 1 décembre 1995

Pagination : $349-358$

ISSN : 1246-8185

Référence électronique

Alain Ginet, "La compréhension et l'expression en environnement multimédia en présentiel et en autonomie guidée », ASp [En ligne], 7-10 | 1995, mis en ligne le 12 novembre 2013, consulté le 03 mai 2019. URL : http://journals.openedition.org/asp/3947 ; DOI : 10.4000/asp.3947

Ce document a été généré automatiquement le 3 mai 2019.

Tous droits réservés 


\title{
La compréhension et l'expression en environnement multimédia en présentiel et en autonomie guidée
}

A piece of cake ou encore $\mathrm{C} 2=\mathrm{E} / \mathrm{M}$, théorisation d'une pratique

\author{
Alain Ginet
}

1 Le titre de cette communication n'est ni une impertinence à l'égard de Monsieur Toubon (chacun sait qu'entrainer les scientifiques à la compréhension n'est pas un morceau de gâteau) ni une impertinence envers Einstein. Il résulte simplement du désir de formuler de manière un peu provocatrice cette constatation que la compréhension et l'expression chez les scientifiques peuvent être considérablement facilitées par un environnement et des supports d'apprentissage multimédia, tout en réduisant considérablement ce que Gillian Brown a appelé « l'effort d'interprétation ».

Plus de vingt ans d'enseignement en Formation continue et près de quinze uniquement à des scientifiques, chercheurs du CNRS ou médecins hospitalo-universitaires, m'ont convaincu du fait que ces adultes nous arrivent en état de traumatisme linguistique, profondément dévalorisés par rapport à leur compétence en langues. Tous vous disent en effet «j'ai fait sept ans d'anglais dans le secondaire, plus deux ou trois dans le supérieur et malgré tout cela je ne sais pas parler ». Les mêmes s'auto-évaluent en général très en dessous de leur performance véritable, persuadés qu'ils sont du caractère gravissime des «fautes » qu'ils commettent par leur passé d'apprenant, où des flots d'encre rouge ont inondé leurs copies. Culpabilisés à l'extrême, ils s'estiment en général mauvais, peu doués, en conformité avec le stéréotype national. Les mêmes vous disent enfin, après une tâche de compréhension sur document audio ou vidéo, "je n'ai rien compris ", alors qu'en général une vérification va faire la preuve qu'ils avaient compris $80 \%$ du message et que seuls quelques mots, expressions ou phrases relevant du détail, leur avaient échappé. Tous vous regardent, du moins au début, avec le plus grand scepticisme lorsque vous leur dites, après démonstration, qu'ils ont en fait bien compris l'essentiel du message. 
3 L'entraînement à la compréhension et le déblocage de la parole avec un public psychologiquement sourd, aveugle et muet ne sont donc pas une mince affaire. Une " affaire » qui comporte en plus une obligation de résultat puisque ces scientifiques ont choisi de venir, malgré les bleus à l'âme de leur passé linguistique. Cette fois, en plus ils paient. Cette fois, c'est donc pour de vrai...

You'd better be good, my dear teacher...

4 Pour ne pas être charlatan (ou démoli à l'heure du bilan), il convient donc de trouver des solutions véritables pour guérir le traumatisme psychologique et remédier à l'inadaptation manifeste des méthodes, des outils, des lieux et des rythmes d'apprentissage.

\section{Guérir le traumatisme}

5 C'est peut-être là la partie la plus difficile. Surtout avec les scientifiques à qui l'on a appris durant toutes leurs études qu'il fallait tout comprendre à $100 \%$; surtout avec les médecins, qui n'ont pas droit à l'erreur. Avec eux tous, pour qui l'incompris est intolérable, ceci d'autant plus que nous, enseignants, leur demandons d'agir... d'agir dans l'instant pour parler, écrire, simuler, présenter, etc., à partir d'une base qu'ils estiment incertaine ou approximative.

$6 \quad$ Il convient donc d'appliquer de manière répétée des stratégies pédagogiques de (re)mise en confiance pour que les apprenants se fassent la preuve à eux-mêmes, de manière mesurable, qu'ils sont bien meilleurs qu'ils ne croyaient pour que l'apprentissage soit gratifiant et valorisant. C'est ce que permettent les documents authentiques et les technologies nouvelles en présentiel et différé. C'est aussi leur faire prendre conscience de leurs stratégies de compréhension et leur apprendre à tolérer l'inconnu (au moins temporairement).

\section{Remédier à l'inadaptation des méthodes et des outils}

7 L'enseignement des langues en France s'est appuyé, s'appuie encore, majoritairement sur l'hémisphère gauche du cerveau analytique et linéaire, siège du langage (parole, lecture, écriture) et du raisonnement logique. En effet, faute de disposer largement de salles de langues équipées pour le visionnement de documents vidéo, et peut-être plus encore faute de formation des enseignants, l'entraînement à la compréhension se fait essentiellement à partir de documents écrits et sonores. Or c'est là utiliser un seul canal, faire prédominer la prise et le traitement de l'information en séquence et en série. C'est donc encourager l'apprenant dans cette idée fausse que la compréhension est un processus rigoureusement linéaire et qu'elle se construit mot à mot. D'où chez lui la ferme croyance en le corollaire que la non-compréhension d'un mot entraîne la noncompréhension du tout, le «je n'ai rien compris» dont nous parlions plus haut. C'est aussi rendre la tâche de compréhension difficile et réductrice dès le départ pour l'apprenant, puisque c'est le priver de toute information visuelle, de toute contextualisation de la langue, de tout le non-verbal d'un échange, et donc d'une grande partie du réel. C'est bien sûr, fractionner aussi l'activation globale du cerveau et réduire considérablement l'aide complémentaire de l'hémisphère droit globalisant et holistique, visuel et synthétique. 
Se priver du visuel dans l'entraînement à la compréhension c'est enfin défavoriser les visuels en faveur des auditifs, les sérialistes en faveur des globalistes faire fi en un mot, de la diversité des profils d'apprentissage mis en lumière par A. de La Garanderie (1980) de la multiplicité des attitudes et stratégies cognitives soulignée par J.-P. Narcy (1990). C'est commettre en quelque sorte ce qu'H. Trocmé (1987: 147) appelle « une faute contre le cerveau ». C'est à coup sûr rendre la tâche très difficile pour les générations présentes d'apprenants qui, enfants de la télévision, ont l'habitude eux, de s'appuyer sur leur hémisphère droit.

9 La première conséquence qui découle de ces remarques est évidente: on ne peut plus aujourd'hui entraîner des apprenants à la compréhension sans partir de documents vidéo authentiques (" authentique » étant pris au sens de « produit pour des natifs ») utilisés de manière active, associés à d'autres supports (audio et/ou textuels) sur le même sujet, travaillés à la fois en collectif, en individuel, en présentiel et en différé. C'est la seule manière d'intégrer les remarques ci-dessus ainsi que les enseignements de la recherche en neuropédagogie et en didactique des langues. À savoir (et entre autres) : le principe d'activation globale du cerveau et d'interpellation totale de la gestion cérébrale (Trocmé $1987: 213)$ et le principe de variation des angles d'approche et des sources d'information pour donner la même information, avec pour objectif final une compréhension multidimensionnelle (Trocmé $1987: 139-40$ et 208).

Il faut ensuite - par référence au filtre affectif que constituent les cerveaux reptilien (blocage possible par réflexe d'autopréservation en langue 2) et limbique, siège de l'émotion et de la mémoire (blocage possible par expérience désagréable) (Narcy 1990 : 40; Trocmé 1987: 47,90) que les documents polymédias en question soient de toute évidence accessibles (tout en restant authentiques, car il est important de se faire la preuve à soi-même que l'on est capable de comprendre en langue étrangère non pas du "fabriqué » à des fins d'apprentissage linguistique, mais du "vrai », conçu pour des natifs), donc gratifiants et motivants. On ira de ce fait de préférence vers l'extra-ordinaire à fort pouvoir d'accroche intellectuelle. Pour l'entrainement de scientifiques à la compréhension en anglais, on peut suggérer l'utilisation d'émissions telles que Horizon ou QED qui, tout en étant de la vulgarisation scientifique, sont d'une très grande qualité en matière d'accroche intellectuelle, de structuration et de prédécoupage didactique, d'utilisation des images comme aides à la compréhension. Ces documents seront associés, selon le cas, à des dossiers de Time Magazine ou Scientific American (par exemple) sur le même sujet et selon un mode qui sera précisé plus loin.

\section{Remédier à l'inadaptation des lieux et des rythmes}

11 La simple salle de cours traditionnelle ne permet pas de prendre en compte tous les paramètres de l'apprentissage et de l'enseignement. Il faut en effet offrir un environnement et des tâches qui permettent d'aller vite et de manière efficace dans l'entraînement à la compréhension, donc d'associer les médias; d'aller loin avec tous, même lorsque les groupes sont hétérogènes (c'est toujours le cas), même lorsque l'assiduité est irrégulière, même lorsque les apprenants arrivent en retard. L'environnement d'apprentissage doit donc offrir la possibilité d'individualisation et de " prise du train en marche »; de démultiplier le temps passé en présentiel puisqu'il est forcément très limité, rarement plus de deux heures hebdomadaires et enfin de prendre 
en compte les processus cognitifs liés au fonctionnement du cerveau. Le processus de compréhension (processus descendant) va en effet du global au détaillé.

langues communicatif analogique, numérique ou mixte. Communicatif signifie que les postes de travail seront disposés en arc de cercle, ou mieux, en tables rondes permettant un travail communicatif en petit groupe et en face à face. Ces tables rondes peuvent s'ouvrir et se déployer en arc de cercle lorsque le travail devient soit collectif (binômes ou grand groupe) soit individuel. Les postes de travail seront dépourvus de cloisons ou, si l'on tient à marquer symboliquement l'isolement à certains moments de la séquence pédagogique, équipés de cloisons mobiles, relevables d'un simple geste. Le matériel choisi devra permettre la mise en communication des apprenants, ou l'entraînement à la communication téléphonique en langue étrangère étant une demande fréquente en Formation continue. À cet égard on se souviendra que le tout informatique n'a permis jusqu'à présent qu'une communication simulée de type radio (où chaque locuteur prend son tour de parole). Ceci dit, depuis Expolangues 95, nous entrons dans une nouvelle ère dans laquelle l'ordinateur équipé d'une carte son remplace définitivement le magnétophone au laboratoire de langues, sans désormais perdre les fonctions communicatives de "pair-work», "conferencing " et téléphone puisqu'on est parvenu enfin à mettre au point une carte spécifique qui permet la superposition des fonctions analogiques et numériques. Le magnétophone audioactif comparatif du passé a donc vécu et se transforme à compter d'aujourd'hui en véritable outil multimédia extraordinairement polyvalent puisque l'ordinateur qui le remplace permet d'associer sur un même poste de travail :

- toutes les fonctions traditionnelles du laboratoire de langues (travail audioactif comparatif tutoré);

- toutes les fonctions communicatives acquises plus récemment (cf. ci-dessus);

- de nouvelles fonctions numériques d'indexation qui permettent à l'apprenant, comme à l'enseignant, de revenir de manière instantanée sur ce qui a posé un problème à un moment quelconque du travail, donc sans rembobinages, recherches ou prises de notes fastidieuses. Le temps de la réécoute et de la répétition linéaires ad nauseam est donc révolu ;

- l'image fixe, importable ou gelable au vol si besoin;

- l'image animée, importable ou « capturable » au vol de même manière ;

- des outils de travail en hypertexte et/ou hypermédia sur réseau.

14 La salle sera équipée de deux écrans vidéo collectifs permettant soit de démultiplier l'image afin que les apprenants puissent la voir dans de bonnes conditions, quelle que soit leur localisation dans la salle; soit de permettre deux affichages différents. On aura par exemple sur l'un la séquence vidéo en cours, et sur l'autre la (les) consigne (s) pour la tâche à effectuer sur la séquence en question.

La salle sera également équipée de plusieurs sources vidéo, au minimum un magnétoscope et un vidéo-présenteur (auxquels on pourra naturellement adjoindre un lecteur (ou une tour) de CDROM, CDV, ou CDI, ou de vidéodisque. L'intérêt du vidéo présenteur, prioritairement aux sources autres que le magnétoscope, est le suivant: caméra vidéo miniature de très haute luminosité (certaines vont jusqu'à 1 lux), montée verticalement sur bras télescopique ou flexible, équipée d'un zoom électrique, elle permet 
de montrer pratiquement n'importe quoi pour tous : objets en trois dimensions, texte et images de livres, transparents, diapositives. Dans la perspective énoncée plus haut d'associer d'autres supports et documents sur le même sujet pour aller vers l'approche multisensorielle préconisée par $\mathrm{H}$. Trocmé, il est évident que cet outil résout une multitude de problèmes matériels que l'enseignant rencontrait jusqu'ici pour montrer. Il offre la couleur en plus, et même une entrée micro si une amplification de la voix du présentateur est nécessaire. Il peut même se transformer enfin, grâce à sa tête pivotante, en caméscope (à poste fixe) ou caméra de surveillance. Outil d'une polyvalence extraordinaire, c'est de ce fait un des meilleurs investissements que l'on puisse faire en matière de nouvelles technologies aujourd'hui.

Le centre d'autoapprentissage guidé, ou une vidéothèque libre-service, sera naturellement le lieu où l'apprenant ira visionner le document vidéo dans son intégralité, puisque, comme on le verra plus loin, il ne lui aura été possible de travailler en cours que sur une très courte séquence. Lui prêter le document lui-même pour travail chez lui serait l'idéal, mais cela est difficilement envisageable, tant pour les problèmes de copyright que de lourdeur de gestion. Le centre d'autoapprentissage sera donc l'extension de la salle de cours multimédia pour continuer à y travailler sa compréhension en différé, à partir de grilles de visionnement destinées à rendre ce visionnement actif et gratifiant, tout en fractionnant une tâche que les moins avancés pourraient juger massive et décourageante. Ceci se fera, bien sûr, dans le temps et au rythme choisi par l'apprenant.

\section{Le déroulement d'un cycle sur un document}

17 Il convient de dire en préambule que le groupe de scientifiques avec lesquels est pratiqué cet entraînement à la compréhension et l'expression a été au préalable testé au moyen de tests informatisés que nous avons mis au point (tests FOCUS ${ }^{1}$ ). Ces tests permettent un bilan de compétence et de performance linguistique individuel. Un listing fait l'état des faiblesses grammaticales, fonctionnelles et notionnelles. Ce diagnostic fait l'objet d'un commentaire individuel. Le même test dégage par ailleurs les dénominateurs communs au groupe et permet de l'ancrer dans un projet collectif autour d'objectifs immédiats ${ }^{2}$. Le groupe, enfin, a été "conscientisé" à sa manière d'apprendre au travers de questionnaires, débattus et commentés ensemble.

Il m'est difficile de présenter ici une approche multimédia puisque je ne dispose pour tout média que de l'écrit. L'exposé ci-après du déroulement d'un cycle sur un document donné ne peut être donc qu'un exemple schématique. Je démarre en général (et nous allons voir pourquoi) la séance initiale par une activité de type spéculatif. Il s'agit souvent d'un montage de trois séquences très brèves, extraites de l'introduction du document vidéo introduction qui, en général, annonce le sujet scientifique traité et sa problématique. Les séquences, que j'ai numérotées au montage, semblent n'avoir aucun rapport entre elles, et fonctionnent donc comme indices dans une enquête dont l'objectif est la découverte du thème. Une des séquences sera par exemple de la vidéo animée, une autre sera purement audio, la troisième une image fixe. La tâche, évidemment, est de décrire pas à pas ce qu'on a vu, entendu, observé, compris, et de formuler des hypothèses sur le thème qui unit les morceaux du puzzle. Le visionnement est collectif, et la discussion se fait en binôme avant toute mise en commun. Il existe une quatrième séquence « joker", plus explicite, dans le cas où les apprenants n'auraient pas découvert le thème, à l'issue du travail sur les trois premières. 
L'intérêt de cette approche est que, simultanément,

- elle s'adresse au cerveau tout entier ;

- elle permet aux visuels de fonctionner sans exclure pour autant les auditifs, et elle les encourage à communiquer ensemble ;

- elle permet la mise en éveil et la mobilisation des facultés de l'individu pour observer, écouter comprendre, en faisant appel à ses acquis linguistiques et expérientiels. La tâche de compréhension est authentique dans la mesure où la résolution du problème ne peut passer que par un rattachement de ce que l'apprenant découvre à ce qu'il sait déjà ${ }^{3}$;

- elle garantit l'accroche intellectuelle ;

- elle enclenche les processus et stratégies cognitifs avant même la découverte du document ;

- elle nécessite de la part des apprenants description ainsi que formulation d'hypothèses, ce qui permet à l'enseignant de faire les apports lexicaux nécessaires de manière naturelle et préalable à l'exercice de compréhension qui va suivre ;

- elle déclenche la communication.

20 C'est donc globalement une " mise en jambes intellectuelle » et une verbalisation qui vont grandement faciliter la compréhension et la rendre gratifiante par la joie de la découverte.

La phase suivante sera la découverte collective de la séquence d'introduction d'où étaient extraits les morceaux du puzzle. Après discussion en binôme, puis collective, de la compréhension globale du thème scientifique et de sa problématique, les apprenants découvrent qu'en même temps qu'ils pratiquaient la compréhension en visionnement collectif, le commentaire audio était enregistré sur leurs magnétophones ou ordinateurs ${ }^{4}$. Ils vont pouvoir maintenant aborder la compréhension de détail, chacun individuellement, à son rythme, en prenant des notes, à la fois pour produire un résumé et pour répondre à des questions. Une mise en commun permet de vérifier l'ensemble et de faire les apports nécessaires. La séance s'achèvera sur la prédiction des techniques scientifiques qui seront adoptées pour essayer d'apporter des réponses aux problèmes que soulève le document.

Les apprenants vont ensuite rentrer chez eux avec pour tâche de lire l'article (un total de 6 pages dont 3 obligatoires) tiré généralement de Time, Scientific American, ou National Geographic, ceci pour le cours suivant, avec pour objectif

- d'y trouver les réponses aux questions de fond posées dans l'introduction du document vidéo ;

- d'écrire un résumé, non pas du contenu du texte lu, mais de ce qui les a le plus intéressés ;

- d'y choisir 10 mots ou expressions en contexte dont ils ont décidé qu'ils leur seraient utiles ;

- de lister par écrit, pour moi l'enseignant, les problèmes de langue ou de civilisation rencontrés, sur lesquels ils souhaitent des éclaircissements ou un apport de connaissances.

Demander autant de travail à des gens aussi occupés était de ma part prendre un pari sur la motivation engendrée, en même temps qu'individualiser et autonomiser l'apprentissage. J'ai le plaisir de dire aujourd'hui, après un an d'expérimentation, que cela a très bien marché. Les chercheurs reviennent en ayant tous lu le texte de très près. Si le résumé n'est pas toujours fait, le choix des mots ou expressions utiles se révèle très intéressant et très enrichissant pour tous. Donner aux apprenants la liberté de construire leur lexique de manière autonome, consciente et raisonnée, leur procure un plaisir extraordinaire et entraîne un niveau de rétention mémorielle bien supérieur. Enfin, 
choisir eux-mêmes ce qu'ils estiment mériter explication ou aide de la part de l'enseignant a le même effet gratifiant.

Tout ce travail de résumé, de compte-rendu, de focalisation sur le lexique et les structures se fera durant le cours suivant, sans qu'on ait à revenir sur l'introduction du document vidéo puisqu'on dispose d'un vidéo-présenteur et de photos, graphiques, dessins ou schémas dans les articles d'accompagnement. Ces illustrations vont en effet servir de support au résumé oral et à la discussion. Même ceux qui n'avaient pu assister au cours précédent, ou qui arrivent en retard, peuvent ainsi "prendre le train en marche » avec une facilité remarquable. Quant au reste du document vidéo - qu'il est bien sûr hors de question de pouvoir visionner ensemble, compte tenu de sa durée (45 minutes en moyenne), l'enseignant en extraira quelques séquences au fil de la discussion pour apporter tel éclairage, tel complément, telle dimension insoupçonnée. La compréhension, même pour les moins avancés, ne posera quasiment aucun problème. Est-il besoin de dire que ceux qui disposent d'un peu de temps courent ensuite voir le document intégral au centre d'autoapprentissage? Ceux, enfin, qui ont fourni un travail écrit peuvent le rendre, bien sûr, pour corrections et remarques.

La troisième séance est consacrée aux apports que l'enseignant juge d'intérêt collectif parmi les demandes individuelles qui se sont exprimées, ainsi qu'à une synthèse collective ou un travail en hypertexte si l'on est équipé d'ordinateurs.

Dans l'idéal le travail en hypertexte et hypermédia se fera dans une quatrième séance collective ou bien en autonomie totale. Grâce à un outil comme Smart Alex $x^{5}$ par exemple, les apprenants pourront en effet effectuer un travail de détail et d'approfondissement extrêmement riche sur le script du document vidéo ou tout document annexe choisi par l'enseignant. Pour aller plus loin encore ils pourront de même procéder à un travail individuel d'enrichissement des aides hypertextuelles en ajoutant leurs propres commentaires, ou en important ce qu'ils seront allés chercher eux-mêmes dans des encyclopédies multimédias sur CDROM aussi extraordinaires qu'Encarta ou Grolier, ou dans des bibliothèques (multimédia elles aussi) telles que Bookshelf94. Ces enrichissements pourront être partagés, après vérification par l'enseignant, en direct ou en différé.

Alors, l'entraînement à la compréhension et l'expression en environnement multimédia ? A piece of cake, littéralement. Facile et gratifiant, un plaisir et une récompense. Pour tous... Pour tous, enseignants et apprenants confondus, réunis par le plaisir d'avoir fait un petit (?) pas ensemble vers la transmission-acquisition d'une langue étrangère, par le plaisir de l'avoir pratiquée beaucoup. Plaisir des apprenants enfin d'avoir recouvré une certaine estime pour eux-mêmes et surtout une confiance grandissante en leurs propres capacités à comprendre et à s'exprimer en langue étrangère.

Une seule ombre au tableau, pour l'instant : le coût de ces équipements, puisqu'un vidéoprésenteur coûte entre 10 et $20000 \mathrm{~F}$, et une salle de cours ou de travail multimédia entre 30 et $35000 \mathrm{~F}$ selon les équipements. C'est beaucoup. En revanche, et si l'on s'y prend bien pour avoir un taux maximal d'utilisation de la salle, la polyvalence des nouveaux matériels permet de diminuer très sensiblement le coût de la salle par apprenant surtout si l'on prend en compte les résultats obtenus. 


\section{BIBLIOGRAPHIE}

Garanderie, A. de la. 1980. Profils pédagogiques. Paris : le Centurion.

Narcy, J.-P. 1990. Apprendre une langue étrangère. Paris : Éditions d'organisation.

Trocmé-Fabre, H. 1987. J'apprends, donc je suis. Paris : Éditions d'organisation.

\section{NOTES}

1. Tests Focus conçus et développés par Jean-Pierre Nougier et Jean Yves Petit Girard 1993, Formation Continue Langues, Université Stendhal, Grenoble.

2. En conformité avec les conditions nécessaires d'acquisition formulées par H. Trocmé (1987 : 132).

3. Cette tâche permet autrement dit le double ancrage dans le présent et dans l'expérience de l'apprenant, qu' H. Trocmé (1987 : 132) considère nécessaires à toute acquisition.

4. Demain, les magnétophones ayant été remplacés par des ordinateurs multimédia puissants, les apprenants disposeront aussi des images et ils pourront revoir la séquence vidéo sans privation médiatique, cérébrale ou communicative.

5. Smart Alex, outil de création d'hypertextes conçu et réalisé par Tony Stenton, Formation Continue Langues, Université Stendhal.

\section{RÉSUMÉS}

Cette communication, théorisation d'une pratique d'enseignement de l'anglais à un vaste public de chercheurs et médecins, tente de vérifier l'hypothèse selon laquelle l'entraînement à la compréhension et à la production orales en langue étrangère est grandement facilité par l'utilisation de supports polymédias dans un environnement nouvelles technologies (permettant à la fois le travail collectif et individuel) en présentiel, et par l'association de tâches en autonomie guidée sur médias associés en temps différé.

This paper is an attempt at theorising our long-standing practise of teaching groups of scientists and medical doctors: our hypothesis is that oral comprehension and production are greatly facilitated by the use of multimedia materials allowing both individual and collective work, in the presence of the teacher and also as guided autonomous work at home or in the work place.

\section{INDEX}

Mots-clés : compréhension, expression, multimédia, nouvelles technologies

Keywords : new technologies 
AUTEUR

ALAIN GINET

Alain Ginet enseigne à l'Université Stendhal - Grenoble 3. 